\title{
ARTICLE
}

Multiple myeloma gammopathies

\section{Pomalidomide, dexamethasone, and daratumumab in relapsed refractory multiple myeloma after lenalidomide treatment}

\author{
David S. Siegel ${ }^{1} \cdot$ Gary J. Schiller $^{2} \cdot$ Christy Samaras $^{3} \cdot$ Michael Sebag $^{4} \cdot$ Jesus Berdeja $^{5} \cdot$ Siddhartha Ganguly $^{6}$. \\ Jeffrey Matous ${ }^{7} \cdot$ Kevin Song $^{8} \cdot$ Christopher S. Seet $^{2} \cdot$ Giampaolo Talamo $^{9} \cdot$ Mirelis Acosta-Rivera $^{10}$ - Michael Bar ${ }^{11}$. \\ Donald Quick ${ }^{12}$ - Bertrand Anz ${ }^{13}$. Gustavo Fonseca ${ }^{14} \cdot$ Donna Reece $^{15} \cdot$ William E. Pierceall $^{16} \cdot$ Weiyuan Chung $^{16}$. \\ Faiza Zafar $^{16} \cdot$ Amit Agarwal $^{16} \cdot$ Nizar J. Bahlis ${ }^{17}$
}

Received: 9 January 2020 / Revised: 6 March 2020 / Accepted: 20 March 2020 / Published online: 6 May 2020

(c) The Author(s) 2020. This article is published with open access

\begin{abstract}
Patients with multiple myeloma who have relapsed after or become refractory to lenalidomide in early treatment lines represent a clinically important population in need of effective therapies. The safety and efficacy of pomalidomide, low-dose dexamethasone, and daratumumab was evaluated in lenalidomide-pretreated patients with relapsed or refractory multiple myeloma (RRMM) after one to two prior treatment lines in the phase 2 MM-014 study. Patients received pomalidomide $4 \mathrm{mg}$ daily from days 1-21 and dexamethasone $40 \mathrm{mg}$ weekly (28-day cycles). Daratumumab $16 \mathrm{mg} / \mathrm{kg}$ was administered per label. Primary endpoint was overall response rate (ORR); secondary endpoints included progression-free survival (PFS) and safety. Per protocol, all patients $(N=112)$ had received lenalidomide in their most recent prior regimen $(75.0 \%$ lenalidomide refractory). ORR was $77.7 \%$ (76.2\% in lenalidomide-refractory patients); median follow-up was 17.2 months. Median PFS was not reached (1-year PFS rate 75.1\%). The most common hematologic grade 3/4 treatment-emergent adverse event was neutropenia (62.5\%). Grade $3 / 4$ infections were reported in $31.3 \%$ of patients, including $13.4 \%$ with grade $3 / 4$ pneumonia. These results demonstrate the safety and efficacy of pomalidomide-based therapy as early as second line in patients with RRMM, even immediately after lenalidomide failure, indicating that switching from the immunomodulatory agent class is not necessary.
\end{abstract}

Supplementary information The online version of this article (https:// doi.org/10.1038/s41375-020-0813-1) contains supplementary material, which is available to authorized users.

David S. Siegel

davids.siegel@hackensackmeridian.org

1 John Theurer Cancer Center, Hackensack University Medical Center, Hackensack, NJ, USA

2 David Geffen School of Medicine at University of California, Los Angeles, CA, USA

3 Cleveland Clinic, Cleveland, OH, USA

4 McGill University Health Centre, Montreal, QC, Canada

5 Sarah Cannon Research Institute, Nashville, TN, USA

6 The University of Kansas Cancer Center, Fairway, KS, USA

7 Colorado Blood Cancer Institute, Denver, CO, USA

8 Vancouver General Hospital, Vancouver, BC, Canada

\section{Introduction}

Advances in therapy have led to improved survival in multiple myeloma (MM); however, nearly every patient will relapse following initial treatment [1-3]. Although most patients will have received frontline treatment with

9 Penn State Hershey Cancer Institute, Hershey, PA, USA

10 Fundación de Investigación, San Juan, PR, USA

11 Stamford Hospital, Stamford, CT, USA

12 Joe Arrington Cancer Research and Treatment Center, Lubbock, TX, USA

13 Tennessee Oncology, Chattanooga, TN, USA

14 Florida Cancer Specialists, St. Petersburg, FL, USA

15 Princess Margaret Cancer Centre, Toronto, ON, Canada

16 Bristol-Myers Squibb, Summit, NJ, USA

17 Arnie Charbonneau Cancer Research Institute, University of Calgary, Calgary, AB, Canada 
lenalidomide-based regimens, patients with lenalidomiderefractory disease have been poorly represented in recent phase 3 relapsed or refractory MM (RRMM) clinical trials [1, 4-9]. Management of these patients remains challenging due to the availability of multiple treatment options combined with factors such as disease aggressiveness, patient age, and response to previous antimyeloma therapies $[3,10]$. In addition, effective treatment of early RRMM is critical because patient outcomes worsen with each relapse, and the interval between relapses shortens with each subsequent line of treatment $[2,3,11]$. Therefore, patients who have become refractory to lenalidomide in early treatment lines are a clinically relevant population in need of proven and effective therapies [1, 4-9].

Pomalidomide, an immunomodulatory agent, exerts potent, direct tumoricidal, and immune-stimulating effects through binding to its molecular target cereblon, a component of the CRL4 E3 ubiquitin ligase, and subsequent degradation of the transcription factors Ikaros and Aiolos $[12,13]$. The antitumor and immune-stimulating properties of pomalidomide are distinct from those of lenalidomide; pomalidomide has different substrate degradation kinetics, increased binding affinity to cereblon, and a different gene modulation profile [12-15]. Pomalidomide also has activity in lenalidomide-resistant myeloma cell lines and animal models and has demonstrated efficacy in patients refractory to lenalidomide in clinical trials $[13,14,16-20]$.

Pomalidomide was initially approved in combination with dexamethasone for the treatment of patients with RRMM and $\geq 2$ prior therapies (including lenalidomide and a proteasome inhibitor in the United States and lenalidomide and bortezomib in the European Union) [21, 22]. More recently, various pomalidomide-based triplet regimens have received regulatory approval. The combination of pomalidomide, dexamethasone, and daratumumab is approved in the United States for the same indication as the doublet regimen, as is the combination of pomalidomide, dexamethasone, and elotuzumab [23, 24]. Findings from the recent phase 3 OPTIMISMM trial that demonstrated a significantly prolonged progression-free survival (PFS) with pomalidomide, bortezomib, and dexamethasone (PVd) vs Vd (11.2 vs 7.1 months; HR, 0.61 [95\% CI, 0.49-0.77]; $p<$ 0.001 ) in patients with one to three prior regimens recently led to approval of PVd in several jurisdictions, including in the European Union and Japan, for the treatment of patients with RRMM who received $\geq 1$ prior regimen [21, 25, 26].

Daratumumab, an anti-CD38 monoclonal antibody, exerts direct on-tumor and immunomodulatory activity [27-30]. Daratumumab was approved in the United States for the treatment of RRMM in combination with pomalidomide and dexamethasone based on results of the phase $1 \mathrm{~b}$ EQUULEUS/MMY1001 RRMM trial [23, 31]. In this study of patients with heavily pretreated RRMM (median, four prior lines of therapy), the triplet combination led to an overall response rate (ORR) of $60 \%$, including an ORR of $58 \%$ in patients who were refractory to both a proteasome inhibitor (PI) and an immunomodulatory agent [31]. Median PFS was 8.8 months, and median overall survival (OS) was 17.5 months. However, this regimen has not been extensively studied in earlier lines of therapy or in patients who became refractory to lenalidomide-based therapy immediately prior to study entry.

The phase $2 \mathrm{MM}-014$ trial investigated the outcomes of sequencing pomalidomide-based therapy immediately after lenalidomide failure in early treatment lines. Here we report safety and efficacy findings from cohort $\mathrm{B}$, in which patients relapsed from or refractory to lenalidomide in their first or second treatment line received pomalidomide, lowdose dexamethasone, and daratumumab.

\section{Methods}

\section{Study design and patients}

MM-014 is a phase 2, nonrandomized, multicenter, openlabel clinical trial with three cohorts conducted at 49 study sites in the United States, Canada, and Japan. Patients in cohort A received pomalidomide plus low-dose dexamethasone. Patients in cohort B received pomalidomide, low-dose dexamethasone, and daratumumab. Cohort $\mathrm{C}$ (currently enrolling) is a Japanese-only arm of the study; patients will receive pomalidomide, low-dose dexamethasone, and daratumumab. Patients were not allocated across cohorts; rather, cohort $\mathrm{B}$ was added to the trial via protocol amendment after the full accrual of cohort $\mathrm{A}$, and cohort $\mathrm{C}$ was added after the full accrual of cohort $\mathrm{B}$.

Patients eligible for inclusion in cohort B of MM-014 were $\geq 18$ years of age with documented MM diagnosis, measurable disease (serum M-protein $\geq 0.5 \mathrm{~g} / \mathrm{dl}$ or urine $\mathrm{M}$ protein $\geq 200 \mathrm{mg} / 24 \mathrm{~h}$ ), and Eastern Cooperative Oncology Group performance status $\leq 2$. In addition, patients were required to have had one or two prior lines of antimyeloma therapy, documented progressive disease (PD) during or after their last line of therapy, and treatment with a lenalidomide-containing regimen for $\geq 2$ consecutive cycles as their most recent regimen. Patients who relapsed after or were refractory to lenalidomide were eligible for inclusion. Refractory disease was defined as nonresponsive to therapy or as PD within 60 days of the last dose.

Key exclusion criteria included prior treatment with pomalidomide or daratumumab or hypersensitivity to thalidomide, lenalidomide, dexamethasone, or monoclonal antibodies. The following laboratory abnormalities were exclusionary: absolute neutrophil count $<1 \times 10^{9} / 1$, platelet count $<75 \times 10^{9} / 1\left(<30 \times 10^{9} / 1\right.$ for patients in whom $\geq 50 \%$ 
of bone marrow nucleated cells were plasma cells), corrected serum calcium $>2.875 \mathrm{mmol} / \mathrm{l}(11.5 \mathrm{mg} / \mathrm{dl})$, hemoglobin $<80 \mathrm{~g} / \mathrm{l}$, aspartate aminotransferase or alanine transaminase $>3.0 \times$ upper limit of normal, serum total bilirubin $>34.2 \mu \mathrm{mol} / \mathrm{l}(2.0 \mathrm{mg} / \mathrm{dl})$ or $3.0 \times$ upper limit of normal, and severe renal impairment (creatinine clearance $<30 \mathrm{ml} / \mathrm{min}$ or requiring dialysis).

\section{Treatment}

Patients received pomalidomide, low-dose dexamethasone, and daratumumab in 28-day cycles until PD or unacceptable toxicity. Both pomalidomide and low-dose dexamethasone were administered orally, and daratumumab was administered intravenously. Pomalidomide $4 \mathrm{mg}$ was given on days 1 to 21 . Dexamethasone $40 \mathrm{mg}(20 \mathrm{mg}$ for patients $>75$ years of age) was given on days $1,8,15$, and 22. Daratumumab $16 \mathrm{mg} / \mathrm{kg}$ was given on days $1,8,15$, and 22 of cycles 1 and 2; days 1 and 15 for cycles 3 through 6; and day 1 for cycle 7 and beyond. Daratumumab dose reductions were not allowed. Thromboprophylaxis was mandatory for all patients and included low-dose aspirin, low-molecular-weight heparin, or other equivalent antithrombotic agents.

Patients experiencing grade 4 neutropenia or febrile neutropenia had their dose of pomalidomide modified. Following an event, the dose was withheld and complete blood counts were followed weekly. If the patient was not already receiving granulocyte-colony stimulating factor (GCSF), the treating physician could initiate G-CSF at their discretion. Absolute neutrophil counts were required to be $\geq 500$ cells $/ \mu$ l prior to restarting pomalidomide.

\section{Endpoints and assessments}

The primary endpoint was ORR. Secondary endpoints were time to response (TTR), duration of response (DOR), PFS, time to progression (TTP), OS, and safety, including adverse events (AEs) and second primary malignancies (SPMs). Exploratory endpoints for cohort B included molecular, immune, and cellular markers potentially predictive of response or resistance to treatment, pharmacodynamic and mechanistic biomarkers, and health-related quality of life (HRQOL).

Tumor response was based on investigator's assessment using local imaging review (if applicable) and central laboratory results according to modified International Myeloma Working Group criteria. Daratumumab-specific serum immunofixation electrophoresis reflex assay was performed per protocol for patients with immunoglobulin- $\gamma$ (IgG) and $-\kappa \mathrm{MM}$ and monoclonal spike of $\leq 0.2 \mathrm{~g} / \mathrm{dl}$. TTR, DOR, TTP, and PFS were calculated based on the investigator's response assessment, and all time-to-event endpoints were estimated from time of study enrollment, except DOR, which was estimated from time of response. Efficacy assessments included myeloma paraprotein, serum immunoglobulins, serum free light chain, bone marrow aspiration and/or biopsy, radiographic assessments of lytic bone lesions, and extramedullary plasmacytoma assessments.

Safety assessments included AEs, physical examination, clinical laboratory evaluations, venous thromboembolism monitoring, and pregnancy testing and counseling. AEs were coded according to Medical Dictionary for Regulatory Activities (version 20.0) and graded according to National Cancer Institute Common Terminology Criteria for Adverse Events (version 4.03). If a patient experienced the same $\mathrm{AE}$ multiple times, only the event of worst severity was counted. SPMs were monitored as events of interest and reported as serious AEs.

HRQOL was assessed via EuroQol EQ-5D at baseline, at day 1 of each treatment cycle prior to treatment administration, and at treatment discontinuation. In addition, the worst change from screening among all postbaseline measurements was analyzed. Overall scores were analyzed using change from baseline assessment at each postbaseline time point using a mixed model with adjustment for baseline covariates.

All patients provided bone marrow aspirate, whole blood, saliva, and normal tissue samples. Baseline and ontreatment peripheral blood samples were collected to evaluate comprehensive immune profiles, including T-cell and NK-cell subset data. All laboratory measures for safety and efficacy assessments were performed centrally, but tests that could result in dose modifications were also performed locally to facilitate treatment-related decisions during patient visits. Local laboratory results were not collected unless specifically requested by the sponsor.

\section{Statistical analysis}

The intention-to-treat (ITT) population, defined as all enrolled patients regardless of whether they received study treatment, was used for all efficacy analyses. The efficacy evaluable (EE) population, comprising enrolled patients who received $\geq 1$ dose of study treatment and had $\geq 1$ postbaseline response assessment, was used to provide supportive sensitivity analyses for ORR, PFS, and OS. The safety population was defined as all enrolled patients who received $\geq 1$ dose of study treatment. The HRQOL-evaluable population comprised patients who had baseline HRQOL evaluation and $\geq 1$ postbaseline evaluation.

Baseline and demographic characteristics were summarized via frequency tabulations for categorical variables and descriptive statistics for continuous variables. For cohort B, a sample size of $\sim 100$ patients was deemed adequate to estimate ORR with a $95 \%$ CI of width $\pm 9.7 \%$ about 
the obtained rate. Point estimates of ORR together with the 95\% CI were calculated using normal approximation to the binomial distribution. Kaplan-Meier procedures were used to characterize time-to-event curves; medians and $95 \% \mathrm{CIs}$ were estimated. Univariate summary statistics were provided for TTR.

\section{Study oversight}

All patients provided written informed consent. This study was carried out in accordance with the principles of the Declaration of Helsinki and the International Conference on Harmonisation's Guideline for Good Clinical Practice as well as applicable local regulations governing conduct of clinical studies. Each participating site's institutional review board or ethics committee approved the study. This study was funded and sponsored by Bristol-Myers Squibb. The study's sponsor compiled and maintained the data. All authors had full access to the data. This study was registered at ClinicalTrials.gov as NCT01946477.

\section{Results}

Cohort B included 112 patients enrolled between September 2016 and December 2017 (Fig. 1). Median age was 66.5 years, and most patients (67.9\%) were male (Table 1). The median time since MM diagnosis was 3.4 years. Per protocol, patients had either one $(n=70 ; 62.5 \%)$ or two prior lines of therapy $(n=42 ; 37.5 \%)$, and all patients were treated with lenalidomide in the immediate prior line of

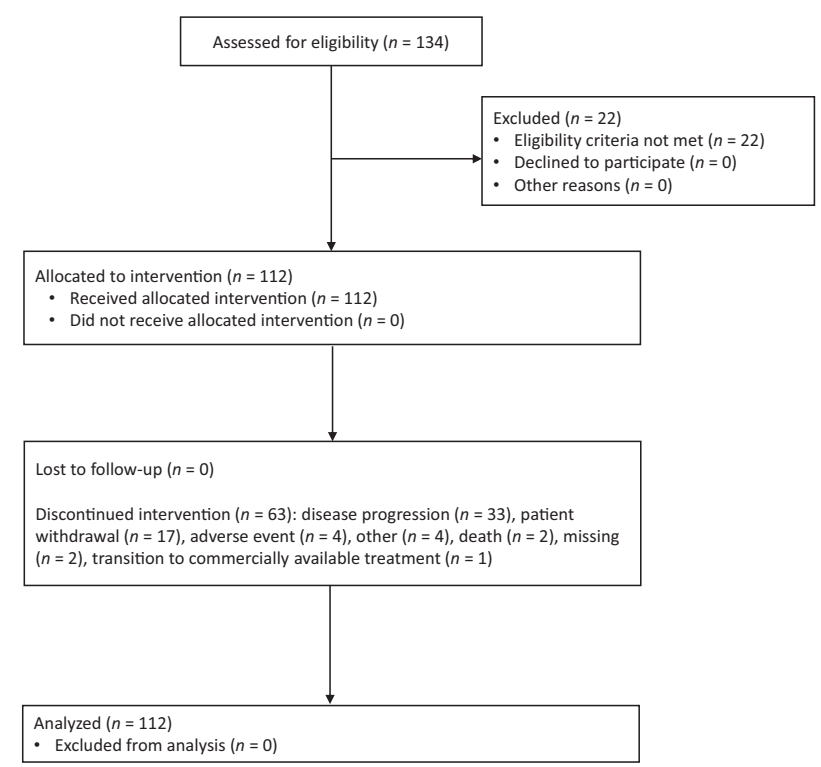

Fig. 1 CONSORT flow diagram of cohort B of MM-014. Cohort B enrolled 112 patients.
Table 1 Demographic and baseline characteristics.

\begin{tabular}{|c|c|}
\hline Characteristic & $\begin{array}{l}\text { ITT population } \\
(N=112)\end{array}$ \\
\hline Age, median (range), years & $66.5(39.0-83.0)$ \\
\hline$>65$ years, $n(\%)$ & $62(55.4)$ \\
\hline Male, $n(\%)$ & $76(67.9)$ \\
\hline \multicolumn{2}{|l|}{ ECOG PS, $n(\%)$} \\
\hline 0 & $44(39.3)$ \\
\hline 1 & $67(59.8)$ \\
\hline 2 & $1(0.9)$ \\
\hline \multicolumn{2}{|l|}{ Calculated R-ISS stage, $n(\%)$} \\
\hline I & $30(26.8)$ \\
\hline II & $53(47.3)$ \\
\hline III & $8(7.1)$ \\
\hline $\mathrm{NE}$ & $21(18.8)$ \\
\hline $\begin{array}{l}\text { Time from MM diagnosis, median } \\
\text { (range), years }\end{array}$ & $3.4(0.5-11.6)$ \\
\hline $\begin{array}{l}\text { Number of prior antimyeloma lines, } \\
\text { median (range) }\end{array}$ & $1(1-2)$ \\
\hline One prior line of therapy, $n(\%)$ & $70(62.5)$ \\
\hline Two prior lines of therapy, $n(\%)$ & $42(37.5)$ \\
\hline \multicolumn{2}{|l|}{ Prior therapies, $n(\%)$} \\
\hline LEN & $112(100)$ \\
\hline Proteasome inhibitor $^{\mathrm{a}}$ & $89(79.5)$ \\
\hline BORT & $87(77.7)$ \\
\hline $\mathrm{CFZ}$ & $11(9.8)$ \\
\hline IXA & $4(3.6)$ \\
\hline Alkylating agents & $89(79.5)$ \\
\hline SCT & $78(69.6)$ \\
\hline Other agents & $8(7.1)^{\mathrm{b}}$ \\
\hline $\begin{array}{l}\text { Refractory to most recent prior LEN- } \\
\text { containing regimen, } n(\%)^{\mathrm{c}}\end{array}$ & $84(75.0)$ \\
\hline $\begin{array}{l}\text { Duration of most recent prior LEN- } \\
\text { containing regimen, median } \\
\text { (range), months }\end{array}$ & $23.9(0.4-116.8)$ \\
\hline \multicolumn{2}{|l|}{ Most recent prior LEN dose, $n(\%)$} \\
\hline $25 \mathrm{mg}$ & $35(31.3)$ \\
\hline $20 \mathrm{mg}$ & $4(3.6)$ \\
\hline $15 \mathrm{mg}$ & $18(16.1)$ \\
\hline$\leq 10 \mathrm{mg}$ & $54(48.2)$ \\
\hline Missing & $1(0.9)$ \\
\hline
\end{tabular}

BORT bortezomib, CFZ carfilzomib, ECOG PS Eastern Cooperative Oncology Group performance status, ITT intention-to-treat, IXA ixazomib, $L E N$ lenalidomide, $M M$ multiple myeloma, $N E$ not evaluable, $R$-ISS revised International Staging System, $S C T$ stem cell transplant.

${ }^{\text {a }}$ Patients may have received $>1$ proteasome inhibitor.

${ }^{\mathrm{b}}$ Including one patient who received doxorubicin, eight patients who received etoposide, and three patients who received cisplatin. Patients may have received $>1$ of these agents.

${ }^{\mathrm{c}}$ Refractoriness to lenalidomide was defined as being refractory to the lenalidomide-containing regimen immediately prior to study entry. 
therapy; 84 patients $(75.0 \%)$ were refractory to lenalidomide. The most recent prior lenalidomide dose was $\leq 10 \mathrm{mg}$ in 54 patients $(48.2 \%)$. Overall, 89 patients $(79.5 \%)$ had prior exposure to a PI; 87 patients $(77.7 \%)$ had received prior treatment with both lenalidomide and bortezomib. Seventy-eight patients $(69.6 \%)$ had undergone prior stem cell transplant. Of 93 patients with available cytogenetic analysis, 73 patients $(78.5 \%)$ were classified as standard risk, and 20 patients $(21.5 \%)$ were classified as high risk (presence of del[17p], t[4;14], and/or t[14;16]).

With a median follow-up of 17.2 months, 63 patients had discontinued treatment at data cutoff (February 8, 2019), and 49 patients remained on active treatment. PD was the most common cause of discontinuation $(n=33)$, followed by patient withdrawal $(n=17)$, AEs $(n=4)$, other reasons $(n=4)$, death $(n=2)$, and transition to another commercially available treatment $(n=1)$; the reason for discontinuation was missing for two patients. Median duration of treatment was 14.6 months for pomalidomide, 13.2 months for low-dose dexamethasone, and 14.4 months for daratumumab (Supplementary Table 1). Median relative dose intensity was $0.9,0.8$, and 1.0 for pomalidomide, lowdose dexamethasone, and daratumumab, respectively.

In the ITT population, 87 patients $(77.7 \%)$ achieved ORR; 57 (50.9\%) achieved very good partial response (VGPR) or better, and 27 patients $(24.1 \%)$ achieved complete response (CR) (Table 2). In the EE population $(n=$ 109), ORR was $79.8 \%$. In both the ITT and EE populations, median TTR was 1.0 month (range, 0.8-4.8 months), and median time to best response was 3.7 months (range, 0.9-20.7 months). For most patients, depth of response increased over time (Fig. 2); 37 patients (42.5\%) did not achieve their best response until $\geq 6$ months. Median DOR

Table 2 Response (mIMWG criteria).

\begin{tabular}{ll}
\hline Response, $n(\%)$ & ITT population $(N=112)$ \\
\hline CBR (MR or better) & $96(85.7)$ \\
ORR (PR or better) & $87(77.7)$ \\
CR & $27(24.1)$ \\
VGPR & $30(26.8)$ \\
PR & $30(26.8)$ \\
MR & $9(8.0)$ \\
SD & $8(7.1)$ \\
PD & $5(4.5)$ \\
NE & $2(1.8)$ \\
Missing & $1(0.9)$ \\
\hline
\end{tabular}

$C B R$ clinical benefit response, $C R$ complete response, ITT intention-totreat, $m I M W G$ modified International Myeloma Working Group, $M R$ minimal response, $N E$ not evaluable, $O R R$ overall response rate, $P D$ progressive disease, $P R$ partial response, $S D$ stable disease, $V G P R$ very good partial response. was not reached in either the ITT or EE population; the 1 -year DOR rate was $77.7 \%$ for both.

In general, ORR among analyzed subgroups was consistent with that of the ITT population (Fig. 3; Supplementary Table 2). The ORR reported in patients with one prior line of therapy $(78.6 \%)$ was similar to that observed in patients with two prior lines of therapy $(76.2 \%)$. Patients who relapsed after or were refractory to lenalidomide had an ORR of $82.1 \%$ and $76.2 \%$, respectively. In patients whose last prior dose of lenalidomide was $\leq 10 \mathrm{mg}$ or $>10 \mathrm{mg}, 85.2 \%$ and $70.2 \%$ achieved ORR, respectively. ORR was $78.7 \%$ in patients who had prior PI and lenalidomide exposure. Patients with standard-risk vs high-risk cytogenetics had an ORR of $79.5 \%$ vs $55.0 \%$, respectively. In addition, ORR was $90.9 \%$ in patients treated with lenalidomide for $>24$ months $(n=55)$ and $64.9 \%$ in patients treated with lenalidomide for $\leq 24$ months $(n=$ 57). Notably, patients with calculated Revised International Staging System stage I disease $(n=30)$ achieved an ORR of $93.3 \%$.

Median PFS was not reached for the ITT (Fig. 4a) or EE populations. The 1-year PFS rates were $75.1 \%$ and $75.9 \%$, respectively. In patients who relapsed after lenalidomide, the 1-year PFS rate was $83.2 \%$, while patients refractory to lenalidomide had a median PFS of 21.8 months and a 1-year PFS rate of $72.4 \%$. Among patients whose last prior dose of lenalidomide was $\leq 10 \mathrm{mg}, 77.9 \%$ remained alive and progression free at 1 year compared with $71.9 \%$ of those whose last prior dose of lenalidomide was $>10 \mathrm{mg}$ (Fig. 4b). In patients with one vs two prior lines of therapy, the 1-year PFS rate was $78.8 \%$ vs $69.0 \%$, respectively (Supplementary Fig. 1). In patients with prior PI and lenalidomide exposure, $75.4 \%$ were alive and progression free at 1 year (Supplementary Fig. 2). The 1-year PFS rate was $82.8 \%$ vs $45.2 \%$ in patients with standard-risk vs high-risk cytogenetics, respectively (Supplementary Fig. 3). In patients whose duration of prior lenalidomide treatment was $>24$ vs $\leq 24$ months, 1-year PFS rates were $85.6 \%$ vs $64.1 \%$, respectively. Median TTP was not reached in either the ITT or EE population. OS data are not yet mature, with 20 deaths documented to date.

Measurable residual disease (MRD) assessments via next-generation sequencing (LymphoTrack, Invivoscribe, San Diego, CA) were performed in 19 patients with best response of VGPR or better and matching screen and response confirmation timepoints. Trackable clones were identified as $>2.5 \%$ of all recombinant clonal sequences from the CDR3 region in 16 of 19 patients (84.2\%); three patients did not have a clone sufficient for indexing. Eight of 16 patients were MRD negative (all $<10^{-4}$ based on cellularity and lowest limit of quantification; three patients had a lower limit of quantification of $10^{-5}$ ). Twelve patients achieved CR; of these patients, seven were MRD negative 
Fig. 2 Depth of response over time in the ITT population ${ }^{\mathrm{a}}$. For response missing $\leq 2$ cycles in the range from the first available response to the last available response, the last response assessment before the gap was carried through the gap. CR complete response, ITT intention-to-treat, MR minimal response, $\mathrm{PD}$ progressive disease, PR partial response, SD stable disease, VGPR very good partial response. ${ }^{a}$ Excludes 1 patient without response. ${ }^{\mathrm{b}}$ Data truncated due to discontinuation, data cutoff, or start of new treatment.

Fig. 3 Overall response rates by subgroups. The vertical line indicates $77.7 \%$, which was the ORR in the overall population. $\mathrm{CrCl}$ creatinine clearance, ECOG PS Eastern Cooperative Oncology Group performance status, HR high-risk, LCL lower control limit, LEN lenalidomide, ORR overall response rate, PI proteasome inhibitor, R-ISS Revised International Staging System, SCT stem cell transplant, SR standard risk, Tx treatment, UCL upper control limit.
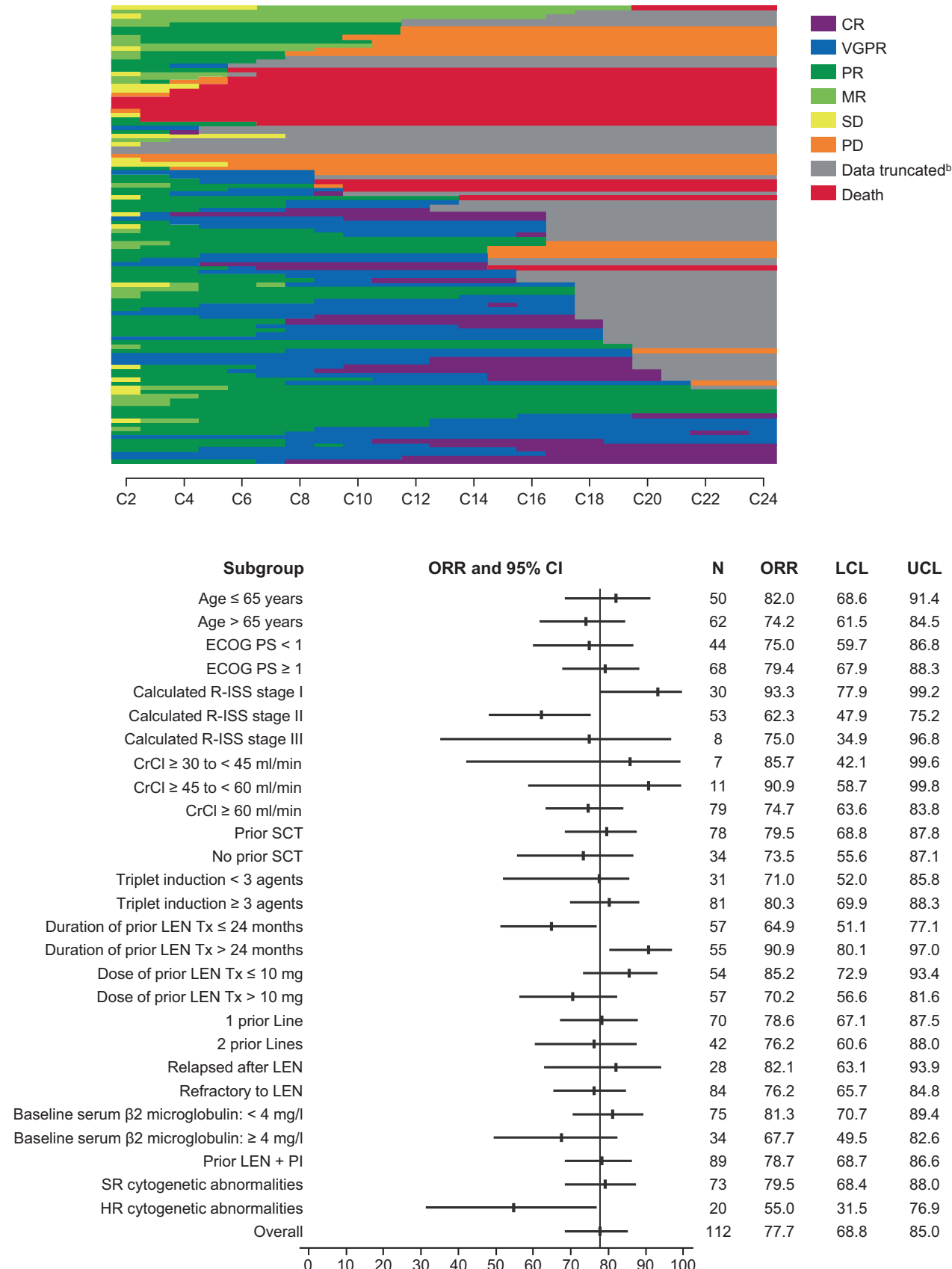

at $\geq 6$ months after study entry. Furthermore, of four patients with VGPR as best response, one patient was MRD negative. All eight MRD-negative patients and seven of eight MRD-positive patients remain in remission, with a minimum follow-up time of 16 months.

Nearly all patients $(99.1 \%)$ had a treatment-emergent adverse event (TEAE). The most common nonhematologic any-grade TEAE was infections and infestations (85 patients [75.9\%]), including $35(31.3 \%)$ with upper respiratory tract infection; the most common hematologic any-grade TEAE was neutropenia (74 patients [66.1\%]). Any-grade infusionrelated reactions were reported in 34 patients $(30.4 \%)$.
The most common grade 3/4 hematologic TEAEs were neutropenia $(62.5 \%)$ and anemia $(17.9 \%)$, and the most common grade $3 / 4$ nonhematologic TEAE was pneumonia (13.4\%) (Table 3). Forty patients (35.7\%) had $\geq 1$ TEAE leading to reduction of pomalidomide (Table 4). The most common TEAE leading to pomalidomide dose reduction was neutropenia (20.5\%). Seventy-eight patients $(69.6 \%)$ had $\geq 1$ TEAE leading to pomalidomide interruption, and $88(78.6 \%)$ had $\geq 1$ TEAE leading to daratumumab interruption. Pomalidomide and daratumumab dose interruptions due to TEAEs were most frequently caused by neutropenia (37.5 and $39.3 \%$ ) and pneumonia (14.3 and 8.9\%). Discontinuations of 


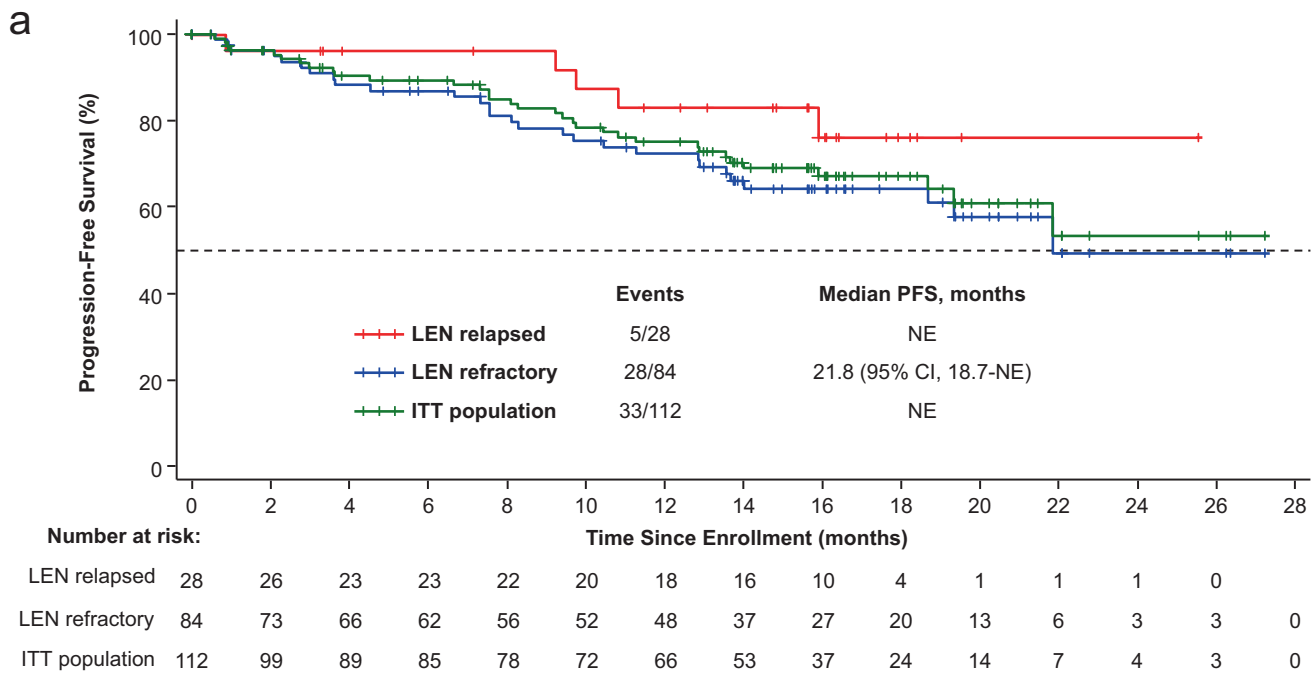

b

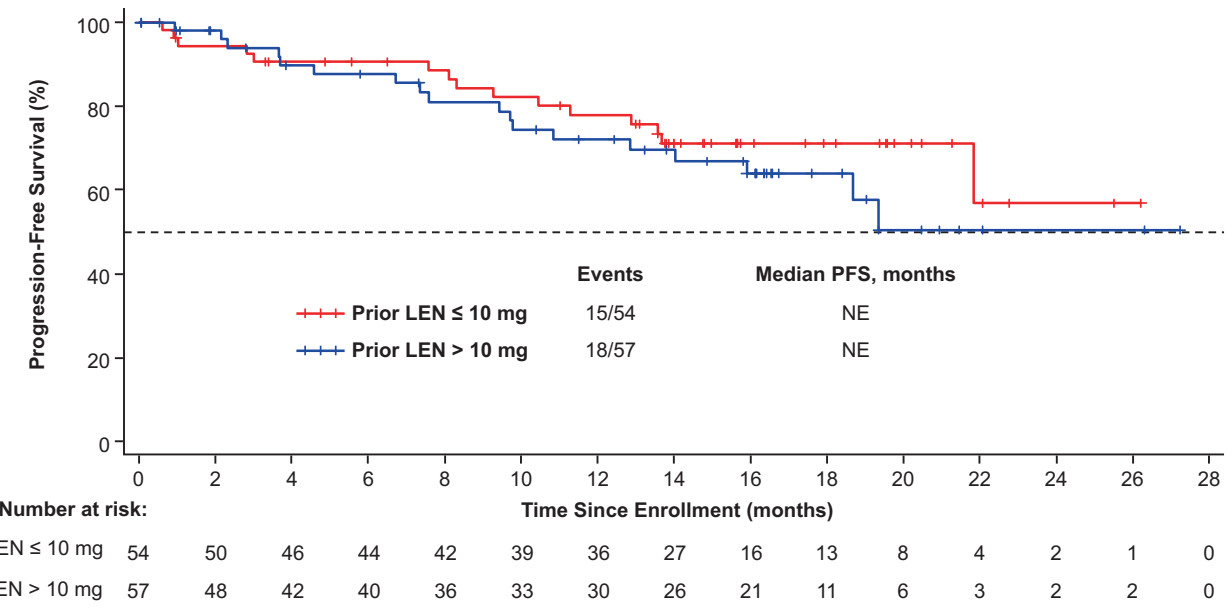

Fig. 4 Progression-free survival. a Median PFS was not reached in the ITT population or in patients who relapsed after lenalidomide. Median PFS was 21.8 months in patients who were refractory to lenalidomide. b Median PFS was not reached in patients whose last prior dose of lenalidomide was $\leq 10 \mathrm{mg}$ or in those whose last prior dose was $>10 \mathrm{mg}$. ITT intention-to-treat, LEN lenalidomide, NE not estimable, PFS progression-free survival.

pomalidomide and daratumumab due to $\geq 1$ TEAE were reported in five and eight patients (4.5\% and $7.1 \%)$, respectively. Six SPMs were reported: two basal cell carcinomas, one metastatic colon cancer, one hepatic cancer, one lymphocytic leukemia, and one squamous cell carcinoma of the skin.

As of 15 October 2018, 108 patients were HRQOL evaluable. EQ-5D completion rates for each evaluated cycle (1-6) were $\geq 88 \%$. Through all six cycles, mean changes from baseline in EQ-5D index and visual analog scale health score were stable. Minimum clinically important improvements in EQ-5D index $(\geq 0.1)$ and visual analog scale health score $(\geq 6)$ were achieved by $28.8 \%$ and $39.0 \%$ of patients at cycle 6 , respectively. EQ-5D index values were stable, with a trend toward improvement in usual activities, pain/discomfort, and anxiety/depression.

\section{Discussion}

The results from cohort B in MM-014 demonstrated the effectiveness and safety of pomalidomide, low-dose dexamethasone, and daratumumab in patients with RRMM immediately after failure of first- or second-line lenalidomide-based treatment. Notably, most patients $(62.5 \%)$ had received only one prior line of therapy, and $75.0 \%$ were lenalidomide refractory. ORR, the primary endpoint, was $77.7 \%$. More than half of patients achieved VGPR or better; responses deepened over time, with $42.5 \%$ of patients reaching their best response after $\geq 6$ months of treatment, indicating the importance of long-term treatment with this regimen. Responses persisted in most patients at 1 year. Half of the patients with VGPR or better and assessable MRD achieved MRD negativity at the lowest limit of 
Table 3 Select grade 3/4 TEAEs.

\begin{tabular}{lc}
\hline TEAEs, $n(\%)^{\mathrm{a}}$ & $\begin{array}{l}\text { Safety population } \\
(N=112)\end{array}$ \\
\hline$\geq 1$ grade 3/4 TEAEs & $104(92.9)$ \\
Grade 3/4 hematologic TEAEs ${ }^{\mathrm{b}}$ & $70(62.5)$ \\
Neutropenia & $11(9.8)$ \\
Febrile neutropenia & $20(17.9)$ \\
Anemia & $14(12.5)$ \\
Thrombocytopenia & $6(5.4)$ \\
Leukopenia & \\
Grade 3/4 nonhematologic TEAEs ${ }^{\mathrm{b}}$ & $35(31.3)$ \\
Infections and infestations & $15(13.4)$ \\
Pneumonia & $5(4.5)$ \\
Influenza & $5(4.5)$ \\
Sepsis & $4(3.6)$ \\
Parainfluenzae virus & $6(5.4)$ \\
Back pain & $6(5.4)$ \\
Insomnia & $5(4.5)$ \\
Atrial fibrillation & $5(4.5)$ \\
Dyspnea & $5(4.5)$ \\
Hyperglycemia & $5(4.5)$ \\
Hypokalemia & $5(4.5)$ \\
Hypertension & $4(3.6)$ \\
Chronic obstructive pulmonary disease & $4(3.6)$ \\
Fatigue & $4(3.6)$ \\
Hypophosphatemia & \\
\hline &
\end{tabular}

TEAE treatment-emergent adverse event.

${ }^{a}$ TEAE severity was graded according to the National Cancer Institute Common Terminology Criteria for Adverse Events version 4.03.

${ }^{b}$ Reported in $\geq 3 \%$ of the safety population.

quantification. At 1 year, $75.1 \%$ of patients were alive and progression free. The reported safety profile was consistent with the known toxicities of the individual agents, and patient HRQOL was either maintained or demonstrated signs of improvement.

Pomalidomide, low-dose dexamethasone, and daratumumab benefitted most evaluated patient subgroups. ORR similar to that of the ITT population was achieved regardless of number of prior lines of therapy (one or two), refractoriness to lenalidomide, or previous bortezomib exposure. Of note, patients whose last prior dose of lenalidomide was $\leq 10 \mathrm{mg}$ derived greater benefit from subsequent pomalidomide therapy than patients whose last prior dose was $>10 \mathrm{mg}$, and patients who were treated with lenalidomide for $>2$ years had improved ORR vs patients who were treated for $\leq 2$ years. More than $50 \%$ of patients with high-risk cytogenetics achieved ORR, indicating activity with this pomalidomide-based regimen in this difficult to treat population; further analyses in patients with high-risk cytogenetics are warranted. Finally, it must be noted that ORRs exceeding 90\% (without the 95\% confidence intervals crossing the ITT ORR of $77.7 \%$ ) were observed in patients with calculated Revised International Staging System I and patients who were treated with lenalidomide for $>2$ years, suggesting that patients with these characteristics could derive the greatest benefit with this regimen.

Despite acquired resistance to lenalidomide in the immediate prior line of therapy, pomalidomide combined with daratumumab and dexamethasone led to deep and durable responses. Findings from the exploratory immune profile analysis demonstrated the benefits of maintaining continuous immunomodulation via sequencing pomalidomide immediately after lenalidomide, even in the context of lenalidomide resistance. The combination of pomalidomide, low-dose dexamethasone, and daratumumab did not impair the innate or adaptive immune compartments and demonstrated significant proliferative activity in CD4, CD8, and NK-cell subsets [32]. The latter finding indicates that pomalidomide-based treatment could offset daratumumabmediated loss of NK cells [30]. Furthermore, changes from baseline, including decreases in naive $\mathrm{T}$ cells and selective increases in proliferative and activated $\mathrm{T}$ cells (with no increase in regulatory $\mathrm{T}$ cells), indicated a shift toward immunocompetence enhancement. Likewise, an exploratory immune profile analysis of cohort A demonstrated that pomalidomide plus low-dose dexamethasone increased $\mathrm{CD}^{+}$and $\mathrm{CD}^{+}{ }^{+}$-cell populations, while $\mathrm{CD} 4^{+} \mathrm{T}$-cell populations remained unchanged [33]. The immune stimulation imparted by pomalidomide-based treatment in patients who relapsed after or became refractory to lenalidomide supports previous data indicating that pomalidomide is pharmacologically distinct from lenalidomide and active in the setting of lenalidomide resistance [12-20]. In addition, the higher 1-year PFS rate and ORR reported in patients who were treated with lenalidomide for $>2$ years vs those treated for $\leq 2$ years, as well as the fact that responses deepened over time, may be suggestive of the benefits of uninterrupted treatment with immunomodulatory agents and tolerance to their related AEs. In clinical practice, physicians may be inclined to change class following PD on or after lenalidomide. However, these findings add to the growing body of evidence indicating that pomalidomide-based regimens, including pomalidomide with daratumumab, can overcome early-line resistance or refractoriness to lenalidomide, demonstrating that there is no evidence-based need to replace an IMiD agent with another drug class after PD on lenalidomide. Recent results with iberdomide, a novel cereblon E3 ligase modulator with enhanced tumoricidal and immunomodulatory activity, further support the beneficial role of continued immunomodulation in MM [34, 35]. 
Table 4 TEAEs leading to dose modifications.

\begin{tabular}{|c|c|c|c|}
\hline \multirow[t]{2}{*}{ TEAEs, $n(\%)$} & \multicolumn{3}{|c|}{ Safety population $(N=112)$} \\
\hline & $\begin{array}{l}\text { Pomalidomide } \\
(n=112)\end{array}$ & $\begin{array}{l}\text { Low-dose } \\
\text { dexamethasone } \\
(n=112)\end{array}$ & $\begin{array}{l}\text { Daratumumab } \\
(n=112)\end{array}$ \\
\hline $\begin{array}{l}\text { Patients with } \geq 1 \text { TEAE leading to } \\
\text { dose reduction }\end{array}$ & $40(35.7)$ & $42(37.5)$ & $-^{b}$ \\
\hline \multicolumn{4}{|l|}{ Hematologic TEAEs ${ }^{\mathrm{a}}$} \\
\hline Neutropenia & $23(20.5)$ & $2(1.8)$ & $-^{b}$ \\
\hline \multicolumn{4}{|l|}{ Nonhematologic TEAEs ${ }^{\mathrm{a}}$} \\
\hline Insomnia & 0 & $11(9.8)$ & $-^{b}$ \\
\hline Hyperglycemia & 0 & $4(3.6)$ & $-^{\mathrm{b}}$ \\
\hline Peripheral edema & 0 & $4(3.6)$ & ${ }^{b}$ \\
\hline $\begin{array}{l}\text { Patients with } \geq 1 \text { TEAE leading to } \\
\text { interruption }\end{array}$ & $78(69.6)$ & $74(66.1)$ & 88 (78.6) \\
\hline \multicolumn{4}{|l|}{ Hematologic TEAEs ${ }^{\mathrm{a}}$} \\
\hline Neutropenia & $42(37.5)$ & $38(33.9)$ & $44(39.3)$ \\
\hline Febrile neutropenia & $6(5.4)$ & $6(5.4)$ & $3(2.7)$ \\
\hline $\begin{array}{l}\text { Neutrophil count } \\
\text { decreased }\end{array}$ & $2(1.8)$ & $2(1.8)$ & $4(3.6)$ \\
\hline Thrombocytopenia & $6(5.4)$ & $5(4.5)$ & $5(4.5)$ \\
\hline Leukopenia & $3(2.7)$ & $3(2.7)$ & $5(4.5)$ \\
\hline \multicolumn{4}{|l|}{ Nonhematologic TEAEs ${ }^{\mathrm{a}}$} \\
\hline Infections & $40(35.7)$ & $36(32.1)$ & $33(29.5)$ \\
\hline Pneumonia & $16(14.3)$ & $13(11.6)$ & $10(8.9)$ \\
\hline $\begin{array}{l}\text { Upper respiratory tract } \\
\text { infection }\end{array}$ & $8(7.1)$ & $7(6.3)$ & $7(6.3)$ \\
\hline Influenza & $6(5.4)$ & $5(4.5)$ & $3(2.7)$ \\
\hline Bronchitis & $4(3.6)$ & $3(2.7)$ & $3(2.7)$ \\
\hline Dyspnea & $4(3.6)$ & $4(3.6)$ & $5(4.5)$ \\
\hline Cough & $3(2.7)$ & $3(2.7)$ & $4(3.6)$ \\
\hline Infusion-related reaction & 0 & 0 & $26(23.2)$ \\
\hline $\begin{array}{l}\text { Patients with } \geq 1 \text { TEAE leading to } \\
\text { discontinuation }^{\text {c }}\end{array}$ & $5(4.5)$ & $8(7.1)$ & $8(7.1)$ \\
\hline
\end{tabular}

TEAE treatment-emergent adverse event.

${ }^{a}$ Reported in $\geq 3 \%$ of patients for any drug. Patients may have had $\geq 1$ TEAE leading to the dose modification. ${ }^{\mathrm{b}}$ Per protocol, daratumumab dose reductions were not allowed.

${ }^{\mathrm{c}}$ All TEAEs leading to discontinuation of each respective drug were reported in $<3 \%$ of patients.
Patients refractory to lenalidomide have been largely excluded from randomized trials evaluating investigational regimens against the standard control regimen of lenalidomide and dexamethasone. However, several phase 3 trials included patients refractory to lenalidomide, such as ENDEAVOR (carfilzomib plus dexamethasone; 25\%), ARROW (once weekly carfilzomib plus dexamethasone; 74\%), OPTIMISMM (PVd; 70\%), CASTOR (daratumumab plus Vd; 28\%), ELOQUENT-3 (elotuzumab, pomalidomide, and dexamethasone; 87\%), and ICARIA (isatuximab, pomalidomide, and dexamethasone; 93\%) [25, 36-44]. CASTOR reported ORR in the subgroup of patients refractory to lenalidomide (at last prior line of therapy); ORR was $80.5 \%$ with daratumumab plus
Vd. ENDEAVOR, OPTIMISMM, and CASTOR reported median PFS values ranging from 8.6 to 9.5 months in lenalidomide-refractory patients who received the investigational treatments. These outcomes are consistent with ORR and median PFS values of the ITT populations from clinical trials that included a high percentage of lenalidomide-refractory patients (e.g., ARROW, OPTIMISMM, ELOQUENT-3, and ICARIA); among patients in the investigational arms, ORR ranged from 53.3 to $82.2 \%$, and median PFS ranged from 10.3 to 11.5 months. While cross-trial comparisons should be interpreted cautiously, the ORR of $76.2 \%$ and 1-year PFS rate of $72.4 \%$ reported in lenalidomide-refractory patients in the current study is encouraging in the context of 
available data from phase 3 RRMM trials that included lenalidomide-refractory patients.

The combination of pomalidomide, low-dose dexamethasone, and daratumumab is currently approved in the United States for the treatment of patients with RRMM and $\geq 2$ prior therapies, including lenalidomide and a PI, based on results of the phase $1 \mathrm{~b}$ MMY1001 trial in heavily pretreated patients with RRMM (median prior lines of therapy, four) $[21,22,31]$. In contrast, patients in our trial were required to have had one or two prior lines of therapy, and $62.5 \%$ of patients had only one prior therapy. It is notable that ORR in this study was higher (77.7 vs $60.2 \%)$ and the rate of grade $3 / 4$ neutropenia was lower (62.5 vs $76.7 \%$ ). Importantly, in the current study, median PFS was not reached, and the 1-year PFS rate was $75.1 \%$, indicating the benefit of initiating this treatment in earlier lines of therapy.

Recently published findings from the daratumumab, carfilzomib, and dexamethasone (DKd) arm of the MMY1001 study and the phase 3 CANDOR study provide valuable context regarding use of daratumumab-containing regimens in this setting [45, 46]. In MMY001, patients with $\operatorname{RRMM}(N=85)$ and a median of two prior lines of therapy, including bortezomib and an immunomodulatory drug, received DKd [45]. Overall, $60 \%$ of patients were lenalidomide refractory. The 1-year PFS rate was $74 \%$ and ORR was $84 \%$. Rates of grade $3 / 4$ neutropenia, thrombocytopenia, and lymphopenia were $21 \%, 31 \%$, and $24 \%$, respectively. Twenty-four patients had cardiac TEAEs, mostly grade $3 / 4$, which improved with carfilzomib interruption. Infusion-related reactions were common (43-60\%). In CANDOR, patients with RRMM and one to three prior lines of therapy were randomized 2:1 to DKd $(n=312)$ or carfilzomib plus dexamethasone (Kd; $n=154)$ [46]. Overall, $42.3 \%$ of patients had previous exposure to lenalidomide and 33\% were refractory to lenalidomide. Median PFS was not reached with DKd vs 15.8 months with $\mathrm{Kd}$, and ORR was $84.3 \%$ vs $74.7 \%(p=0.00040)$. Grade $\geq 3$ cardiac failure was reported in $3.9 \%$ and $8.5 \%$ of patients receiving $\mathrm{DKd}$ and $\mathrm{Kd}$, respectively.

Despite the higher proportion of lenalidomide-refractory patients in cohort B of MM-014, key efficacy outcomes between DKd in the MMY1001 and CANDOR trials and pomalidomide, low-dose dexamethasone, and daratumumab were similar. In addition, while the rate of grade 3/4 neutropenia in the present study was higher than in MMY1001, rates of grade 3/4 thrombocytopenia and lymphopenia were lower. Notably, infusion-related reactions also occurred less frequently in the present study (30.4\%) than in MMY1001. Taken together with results from cohort $\mathrm{B}$, these findings demonstrate that the pomalidomide, low-dose dexamethasone, and daratumumab regimen is an effective treatment option in the setting of lenalidomide-refractory disease. Findings from ongoing phase 3 trials evaluating pomalidomide-based regimens with monoclonal antibodies will provide further context. Interim results of the ICARIA trial evaluating pomalidomide, low-dose dexamethasone, and isatuximab in a 100\% lenalidomide-exposed and $93 \%$ lenalidomide-refractory patient population (median, three prior lines of therapy) demonstrated significantly longer PFS (median, 11.5 vs 6.5 months; $p=0.001$ ) and significantly higher ORR $(60.4 \%$ vs $35.3 \% ; p<0.0001)$ compared with pomalidomide and low-dose dexamethasone [43, 44].

In clinical practice, physicians may be inclined to switch away from the immunomodulatory agent drug class after lenalidomide-based treatment failure. These findings demonstrate that pomalidomide, low-dose dexamethasone, and daratumumab is a safe and effective treatment for patients with RRMM immediately following disease progression on or after lenalidomide. These data also continue to support the integration of pomalidomide-based regimens as early as second line in the management of RRMM, even in patients refractory to lenalidomide.

Acknowledgements The authors would like to thank our patients and their families, in addition to the study investigators, participating sites, nurses, and study personnel. The authors would also like to thank Yingdong Lu for statistical programming support, John Arzadon and Natalya Serbina for assistance with data review, Fadi Towfic for assistance with cytogenetic risk assessment, and Jorge Mouro for study support. The authors also acknowledge Shawn Vahabzadeh of MediTech Media, Ltd, for medical writing assistance in the preparation of this manuscript, funded by Bristol-Myers Squibb. This study was funded and sponsored by Bristol-Myers Squibb. The study sponsor and all authors accept full responsibility for the accuracy and completeness of the data.

Author contributions All authors have contributed to the acquisition, analysis, or interpretation of data for this article, contributed to drafts of the article, revised the manuscript critically for important intellectual content, approved the final version to be published, and agreed to be accountable for all aspects of the article.

\section{Compliance with ethical standards}

Conflict of interest DSS reports speakers bureau for Celgene, a Bristol-Myers Squibb company, Amgen, Takeda, Merck, and Novartis; GJS reports research funding from Celgene, a Bristol-Myers Squibb company; CSS, CS, GT, DQ, MA-R, and BA have nothing to disclose; MS reports consultancy for Celgene, a Bristol-Myers Squibb company, and Janssen; JB reports research funding from Abbvie, Amgen, Acetylon, Bluebird, Bristol-Myers Squibb, Celgene, a BristolMyers Squibb company, Constellation, CURIS, Genentech, Glenmark, Janssen, Kesios, Lilly, Novartis, Poseida, Sanofi, Takeda, Teva, Vivolux and consultancy from Takeda, Bristol-Myers Squibb, Karyopharm, CRISPR Therapeutics, Celgene, a Bristol-Myers Squibb company, Kite Pharma Inc, Servier, Janssen, Amgen, BioClinica, Prothena; SG reports speakers bureau for Seattle Genetics; JM reports consultancy for, honoraria from, and speakers bureau for Celgene, a Bristol-Myers Squibb company; KS reports honoraria and research funding from Celgene, a Bristol-Myers Squibb company, Janssen, Amgen, Takeda; MB reports consultancy for Celgene, a Bristol-Myers Squibb company; GF reports speakers bureau for Celgene, a Bristol- 
Myers Squibb company; DR reports consultancy for and honoraria and research funding from Janssen, Celgene, a Bristol-Myers Squibb company, Amgen, Takeda, serving on board of directors or advisory committee for Janssen and Celgene, a Bristol-Myers Squibb company, honoraria and research funding from Merck, and research funding from Otsuka; WEP, WC, and AA report employment with and equity ownership in Bristol-Myers Squibb; FZ reports employment with Bristol-Myers Squibb; NJB reports consultancy for, honoraria and research funding from, and speakers bureau for Celgene, a BristolMyers Squibb company, consultancy for Amgen, and speakers bureau, consultancy for, and research funding from Johnson \& Johnson.

Publisher's note Springer Nature remains neutral with regard to jurisdictional claims in published maps and institutional affiliations.

Open Access This article is licensed under a Creative Commons Attribution 4.0 International License, which permits use, sharing, adaptation, distribution and reproduction in any medium or format, as long as you give appropriate credit to the original author(s) and the source, provide a link to the Creative Commons license, and indicate if changes were made. The images or other third party material in this article are included in the article's Creative Commons license, unless indicated otherwise in a credit line to the material. If material is not included in the article's Creative Commons license and your intended use is not permitted by statutory regulation or exceeds the permitted use, you will need to obtain permission directly from the copyright holder. To view a copy of this license, visit http://creativecommons. org/licenses/by/4.0/.

\section{References}

1. Moreau $\mathrm{P}$, de Wit E. Recent progress in relapsed multiple myeloma therapy: implications for treatment decisions. Br J Haematol. 2017; 179:198-218.

2. Harousseau JL, Attal M. How I treat first relapse of myeloma. Blood. 2017;130:963-73.

3. Sonneveld P, Broijl A. Treatment of relapsed and refractory multiple myeloma. Haematologica. 2016;101:396-406.

4. Raab MS, Cavo M, Delforge M, Driessen C, Fink L, Flinois A, et al. Multiple myeloma: practice patterns across Europe. Br J Haematol. 2016;175:66-76.

5. Moreau P, Zamagni E, Mateos M-V. Treatment of patients with multiple myeloma progressing on frontline-therapy with lenalidomide. Blood Cancer J. 2019;9:38.

6. Lonial S, Dimopoulos M, Palumbo A, White D, Grosicki S, Spicka I, et al. Elotuzumab therapy for relapsed or refractory multiple myeloma. N Engl J Med. 2015;373:621-31.

7. Stewart AK, Rajkumar SV, Dimopoulos MA, Masszi T, Špička I, Oriol A, et al. Carfilzomib, lenalidomide, and dexamethasone for relapsed multiple myeloma. N Engl J Med. 2015;372:142-52.

8. Moreau P, Masszi T, Grzasko N, Bahlis NJ, Hansson M, Pour L, et al. Oral ixazomib, lenalidomide, and dexamethasone for multiple myeloma. N Engl J Med. 2016;374:1621-34.

9. Dimopoulos MA, Oriol A, Nahi H, San-Miguel J, Bahlis NJ, Usmani SZ, et al. Daratumumab, lenalidomide, and dexamethasone for multiple myeloma. N Engl J Med. 2016;375:1319-31.

10. Cornell RF, Kassim AA. Evolving paradigms in the treatment of relapsed/refractory multiple myeloma: increased options and increased complexity. Bone Marrow Transpl. 2016;51:479-91.

11. Yong K, Delforge M, Driessen C, Fink L, Flinois A, GonzalezMcQuire S, et al. Multiple myeloma: patient outcomes in realworld practice. Br J Haematol. 2016;175:252-64.

12. Bjorklund CC, Lu L, Kang J, Hagner PR, Havens CG, Amatangelo $\mathrm{M}$, et al. Rate of CRL4(CRBN) substrate Ikaros and
Aiolos degradation underlies differential activity of lenalidomide and pomalidomide in multiple myeloma cells by regulation of cMyc and IRF4. Blood Cancer J. 2015;5:e354.

13. Lopez-Girona A, Mendy D, Ito T, Miller K, Gandhi AK, Kang J, et al. Cereblon is a direct protein target for immunomodulatory and antiproliferative activities of lenalidomide and pomalidomide. Leukemia. 2012;26:2326-35.

14. Ocio EM, Fernández-Lázaro D, San-Segundo L, López-Corral L, Corchete LA, Gutiérrez NC, et al. In vivo murine model of acquired resistance in myeloma reveals differential mechanisms for lenalidomide and pomalidomide in combination with dexamethasone. Leukemia. 2014;29:705-14.

15. Sehgal K, Das R, Zhang L, Verma R, Deng Y, Kocoglu M, et al. Clinical and pharmacodynamic analysis of pomalidomide dosing strategies in myeloma: impact of immune activation and cereblon targets. Blood. 2015;125:4042-51.

16. Dimopoulos MA, Palumbo A, Corradini P, Cavo M, Delforge M, Di Raimondo F, et al. Safety and efficacy of pomalidomide plus low-dose dexamethasone in STRATUS (MM-010): a phase 3b study in refractory multiple myeloma. Blood. 2016;128:497-503.

17. Leleu X, Attal M, Arnulf B, Moreau P, Traulle C, Marit G, et al. Pomalidomide plus low-dose dexamethasone is active and well tolerated in bortezomib and lenalidomide-refractory multiple myeloma: Intergroupe Francophone du Myélome 2009-02. Blood. 2013;121:1968-75.

18. Richardson PG, Siegel DS, Vij R, Hofmeister CC, Baz R, Jagannath $\mathrm{S}$, et al. Pomalidomide alone or in combination with low-dose dexamethasone in relapsed and refractory multiple myeloma: a randomized phase 2 study. Blood. 2014;123:1826-32.

19. Rychak E, Mendy D, Shi T, Ning Y, Leisten J, Lu L, et al. Pomalidomide in combination with dexamethasone results in synergistic anti-tumour responses in pre-clinical models of lenalidomide-resistant multiple myeloma. Br J Haematol. 2016; 172:889-901.

20. Miguel JS, Weisel K, Moreau P, Lacy M, Song K, Delforge M, et al. Pomalidomide plus low-dose dexamethasone versus highdose dexamethasone alone for patients with relapsed and refractory multiple myeloma (MM-003): a randomised, open-label, phase 3 trial. Lancet Oncol. 2013;14:1055-66.

21. Imnovid (pomalidomide) [summary of product characteristics]. Utrecht, the Netherlands: Celgene Europe B.V.; 2019.

22. Pomalyst (pomalidomide) [package insert]. Summit, NJ: Celgene Corporation; 2019.

23. Darzalex (daratumumab) [package insert]. Horsham, PA: Janssen Biotech, Inc.; 2019.

24. Empliciti (elotozumab) [package insert]. Princeton, NJ: BristolMyers Squibb Company; 2019.

25. Richardson PG, Oriol A, Beksac M, Liberati AM, Galli M, Schjesvold F, et al. Pomalidomide, bortezomib, and dexamethasone for patients with relapsed or refractory multiple myeloma previously treated with lenalidomide (OPTIMISMM): a randomised, openlabel, phase 3 trial. Lancet Oncol. 2019;20:781-94.

26. Pomalyst (pomalidomide) [package insert]. Tokyo, Japan: Celgene KK; 2019.

27. de Weers M, Tai YT, van der Veer MS, Bakker JM, Vink T, Jacobs DC, et al. Daratumumab, a novel therapeutic human CD38 monoclonal antibody, induces killing of multiple myeloma and other hematological tumors. J Immunol. 2011;186:1840-8.

28. Overdijk MB, Verploegen S, Bogels M, van Egmond M, Lammerts van Bueren JJ, Mutis T, et al. Antibody-mediated phagocytosis contributes to the anti-tumor activity of the therapeutic antibody daratumumab in lymphoma and multiple myeloma. MAbs. 2015;7:311-21.

29. Krejcik J, Casneuf T, Nijhof IS, Verbist B, Bald J, Plesner T, et al. Daratumumab depletes CD38+ immune regulatory cells, promotes 
T-cell expansion, and skews T-cell repertoire in multiple myeloma. Blood. 2016;128:384-94.

30. Casneuf T, Xu XS, Adams HC 3rd, Axel AE, Chiu C, Khan I, et al. Effects of daratumumab on natural killer cells and impact on clinical outcomes in relapsed or refractory multiple myeloma. Blood Adv. 2017;1:2105-14.

31. Chari A, Suvannasankha A, Fay JW, Arnulf B, Kaufman JL, Ifthikharuddin JJ, et al. Daratumumab plus pomalidomide and dexamethasone in relapsed and/or refractory multiple myeloma. Blood. 2017;130:974-81.

32. Pierceall WE, Bahlis N, Siegel DS, Schiller GJ, Samaras CJ, Sebag M, et al. Immune profiling of relapsed or refractory multiple myeloma patients treated with pomalidomide and low-dose dexamethasone in combination with daratumumab. Blood. 2018; 132:Abstract 2012.

33. Siegel DS, Schiller GJ, Song KW, Agajanian R, StockerlGoldstein K, Kaya H, et al. Pomalidomide plus low-dose dexamethasone in relapsed refractory multiple myeloma after lenalidomide treatment failure. Br J Haematol. 2020;188:501-10.

34. Lonial S, van de Donk NWCJ, Popat R, Zonder JA, Minnema MC, Larsen J, et al. First clinical (phase 1b/2a) study of iberdomide (CC220; IBER), a CELMoD, in combination with dexamethasone (DEX) in patients (pts) with relapsed/refractory multiple myeloma (RRMM). J Clin Oncol. 2019;37:Abstract 8006.

35. Bjorklund CC, Kang J, Amatangelo M, Polonskaia A, Katz M, Chiu $\mathrm{H}$, et al. Iberdomide (CC-220) is a potent cereblon E3 ligase modulator with antitumor and immunostimulatory activities in lenalidomide- and pomalidomide-resistant multiple myeloma cells with dysregulated CRBN. Leukemia. 2020;34:1197-201.

36. Dimopoulos MA, Moreau P, Palumbo A, Joshua D, Pour L, Hájek $\mathrm{R}$, et al. Carfilzomib and dexamethasone versus bortezomib and dexamethasone for patients with relapsed or refractory multiple myeloma (ENDEAVOR): a randomised, phase 3, open-label, multicentre study. Lancet Oncol. 2016;17:27-38.

37. Moreau P, Joshua D, Chng WJ, Palumbo A, Goldschmidt H, Hájek R, et al. Impact of prior treatment on patients with relapsed multiple myeloma treated with carfilzomib and dexamethasone vs bortezomib and dexamethasone in the phase 3 ENDEAVOR study. Leukemia. 2017;31:115-22.

38. Moreau P, Mateos MV, Berenson JR, Weisel K, Lazzaro A, Song $\mathrm{K}$, et al. Once weekly versus twice weekly carfilzomib dosing in patients with relapsed and refractory multiple myeloma (A.R.R.O. W.): interim analysis results of a randomised, phase 3 study. Lancet Oncol. 2018;19:953-64.

39. Palumbo A, Chanan-Khan A, Weisel K, Nooka AK, Masszi T, Beksac M, et al. Daratumumab, bortezomib, and dexamethasone for multiple myeloma. N Engl J Med. 2016;375:754-66.

40. Spencer A, Hungria VTM, Mateos M-V, Nooka A, Estell J, Barreto WG, et al. Daratumumab, bortezomib, and dexamethasone (DVd) versus bortezomib and dexamethasone $(\mathrm{Vd})$ in relapsed or refractory multiple myeloma (RRMM): updated efficacy and safety analysis of Castor. Blood. 2017;130: Abstract 3145.

41. Spencer A, Lentzsch S, Weisel K, Avet-Loiseau H, Mark TM, Spicka I, et al. Daratumumab plus bortezomib and dexamethasone versus bortezomib and dexamethasone in relapsed or refractory multiple myeloma: updated analysis of CASTOR. Haematologica. 2018;103:2079-87.

42. Dimopoulos MA, Dytfeld D, Grosicki S, Moreau P, Takezako N, Hori M, et al. Elotuzumab plus pomalidomide and dexamethasone for multiple myeloma. N Engl J Med. 2018;379:1811-22.

43. Richardson PG, Attal M, Campana F, Le-Guennec S, Hui AM, Risse ML, et al. Isatuximab plus pomalidomide/dexamethasone versus pomalidomide/dexamethasone in relapsed/refractory multiple myeloma: ICARIA Phase III study design. Future Oncol. 2018;14:1035-47.

44. Richardson PG, Attal M, Rajkumar SV, San Miguel J, Beksac M, Spicka I, et al. A phase III randomized, open label, multicenter study comparing isatuximab, pomalidomide, and low-dose dexamethasone versus pomalidomide and low-dose dexamethasone in patients with relapsed/refractory multiple myeloma (RRMM). J Clin Oncol. 2019;37:Abstract 8004.

45. Chari A, Martinez-Lopez J, Mateos MV, Bladé J, Benboubker L, Oriol A, et al. Daratumumab plus carfilzomib and dexamethasone in patients with relapsed or refractory multiple myeloma. Blood. 2019;134:421-31.

46. Usmani SZ, Quach H, Mateos M-V, Landgren O, Leleu X, Siegel DS, et al. Carfilzomib, dexamethasone, and daratumumab versus carfilzomib and dexamethasone for the treatment of patients with relapsed or refractory multiple myeloma (RRMM): primary analysis results from the randomized, open-label phase 3 study CANDOR (NCT03158688). Blood. 2019;134:Abstract LBA-6. 